\title{
The vertebrate fauna along the Hantangang (river) of Korea
}

\author{
Yeong-Seok Jo${ }^{1}$, Byung-Jik Kim², Wee-Haeng Hur $^{2}$ and Chang-Man Won ${ }^{3, *}$ \\ ${ }^{1}$ Research Cooperation Division, National Institute of Biological Resources, Incheon 404-170, Korea \\ ${ }^{2}$ Animal Resources Division, National Institute of Biological Resources, Incheon 404-170, Korea \\ ${ }^{3}$ Exhibition and Education Division, National Institute of Biological Resources, Incheon 404-170, Korea \\ *Correspondent: wonkorea@chol.com
}

\begin{abstract}
Before the construction of Hantangang dam, we surveyed vertebrate fauna along the Hantangang River and collected samples. We collected 248 specimens (1,394 individuals) of 41 fish species from 10 families in four orders, including 17 endemic species and 5 endangered species. In our herpetofauna survey, we collected 47 amphibian samples of 10 species from 5 families in 2 orders, and 19 reptile samples of 5 species from 2 families in 1 order. We observed 25 species of bird (6 orders, 14 families) inclulding collection of 51 bird specimens (10 species from 5 families in 1 order). We collected 91 mammals of 6 species, and identified 7 species from field sign and direct observation from a total of 10 families in 5 orders. This collection and list comprise the final vertebrate faunal survey of the Hantangang region, and will provide basic scientific information for future research on conservation and restoration of the region.
\end{abstract}

Keywords: amphibians, birds, fauna, fishes, Hantangang, mammals, reptiles

\section{INTRODUCTION}

Habitat fragmentation or reduction by anthropogenic disturbance has been globally regarded as a main cause of extinction (Van Dyke, 2008). Building dams may have direct and indirect effects on local fauna (McCully, 2001). Since World War II and Korean War, wildlife habitats on the Korean peninsula have been drastically altered (Won and Smith, 1999). Even though the armistice of the Korean War was made in 1953, there have been several skirmishes between Repulic of Korea and Democratic People's Republic of Korea. Due to threats of heavy water flow by North Korea and flood control, Hantangang dam was planned to be built despite the surrounding environmental issues. The Hantangang River headwaters are in Pyeonggang-gun, Gangwon-do, North Korea, and the river flows through Gangwon-do and Gyeonggi-do (Fig. 1) and volcanic pleatue covers most of the riparian area (Won et al., 2010). Even though the river is a type locality for Hanta virus found in Striped Field Mouse (Apodemus agrarius) from Hantangang population (Johnson, 2001), the fauna of this river has not been undergone scientific survey. Dam construction commenced in 2007, and was scheduled for completion in 2015 , at which point $14.6 \mathrm{~km}^{2}$ of riparian habitat were expected to be submerged by a lake, and consequent microclimate changes were predicted.

Relatively lagrge vertebrate species are regarded as charismatic animals, and therefore, these taxa have been appreciated as umbrella species for ecosystem (Van Dyke,
2008). Since every toxon survey can be carried out due to economic issue, vertebrate faunal survey has to be prior to other taxa (Tucker, 2005).

We surveyed the area's vertebrate fauna prior to the alteration of riparian habitat along the Hantangang River and collected vertebrate specimens. This would be the last faunal investigation along the Hantangang River. We ascertain that this survey list and collections can provide scientific information for conservation and restoration of the region.

\section{Methods and Materials}

\section{Fishes}

We caught fishes along Hantangang in Cheorwon-gun, Yeoncheon-gun and Pocheon-si from April, July, October and November in 2008.

We used kick nets or cast nets for collecting fishes, and the fishes were immediately fixed with $10 \%$ formalin solution in the field, and preserved in $75 \%$ ethyl alcohol solution for long-term preservation (NIBR, 2007). All specimens collected for this research were registered and deposited in the National Institute of Biological Resources, Korea.

The species are listed alphabetically within the families, which have been arranged according to Nelson (2006). Scientific names are generally those of Kim et al. (2005) with some modification by recent taxonomic works. 


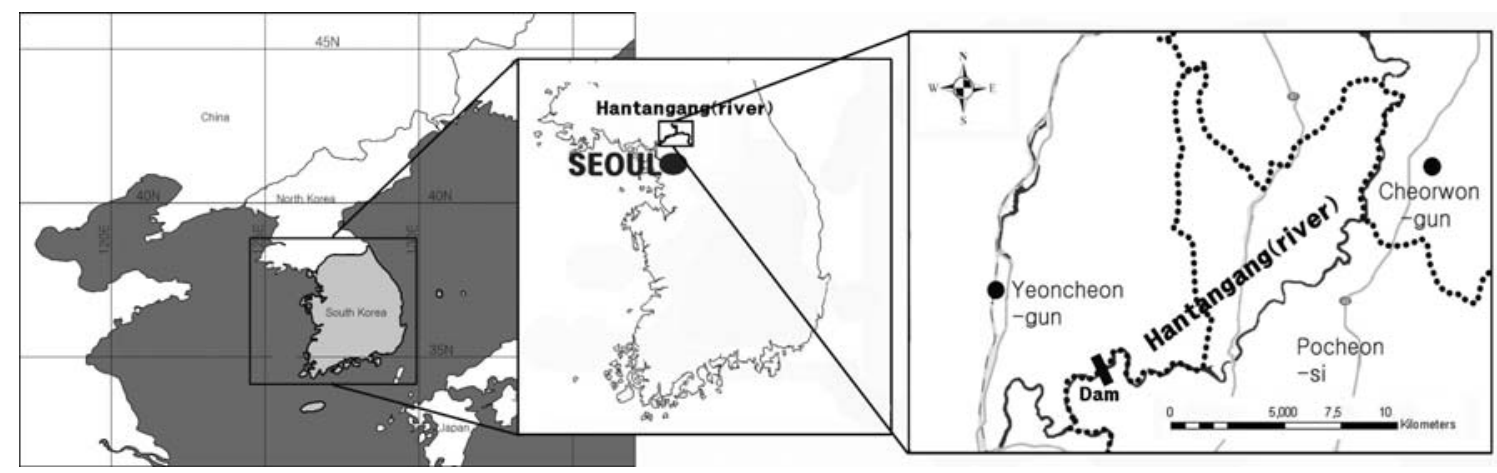

Fig. 1. Maps denoting Hantangang (river) where the survey were done.

\section{Amphibians and reptiles}

For this herpetofauna survey, we caught amphibians and reptiles from March to October 2008 along Hantangang of Cheorwon-gun, Yeoncheon-gun and Pocheonsi. Kick net, hand net and snake tongs were used for colleting amphibians and reptiles. Collected samples were immediately fixed with $10 \%$ formalin solution or $70-80 \%$ ethyl alcohol solution in the field and preserved in $75 \%$ ethyl alcohol solution for long-term preservation. We followed NIBR protocol for making specimens (NIBR, 2007). All specimens collected for this survey were registered and deposited in the National Institute of Biological Resources, Korea.

\section{Birds}

We investigated avian fauna in riparian vegetation, river banks, creeks and shrub communities along Hangtangang (Yeoncheon-gun and Pocheon-si) using mist nets on February, May and October of 2008. Mist nets (height $2.6 \mathrm{~m}$ $\times$ length $12 \mathrm{~m}, 4$ strata) were made of nylon with a mesh size of $36 \mathrm{~mm}$. While mist nets were set up, we observed bird directly through binoculars.

Collected birds were kept below $-20^{\circ} \mathrm{C}$ and made as study skins according to NIBR protocol (NIBR, 2007). All specimens collected for this research were registered and deposited in the National Institute of Biological Resources, Korea.

\section{Mammals}

For mammal collection, we selected trapping sites according to possibility of approach and submergence by dam. We caught small mammals in March, June and October of 2008 and set $\geq 10$ to 30 traps in grid or linear system for 5 successive nights. We used kill traps (Victor professional rat trap) and live traps (Sherman live trap) for this collection, and baited traps with peanut butter (Jones et al., 1996).

All samples, except those from the last day of trapping, were skinned and prepared as study skin in the field on each trapping day according to NIBR protocol (NIBR, 2007). The samples caught on the final trapping day were kept in a cooler with ice and prepared as study skins in the laboratory in one week.

We got permissions for this collection by local government and the Han River Watershed Management Office.

\section{Results AND Discussion}

\section{Fishes}

Along the Hantangang, we collected and identified 248 specimens (1,394 individuals) of 41 species from 10 families in 4 orders (Table 1). As 186 specimens were collected from 28 species, the family Cyprinidae was the most abundant taxon, followed by Cobitidae (22 specimens of 4 speices), Centropomidae (8 specimens of 2 speices), Odontobutidae (11 specimens of 1 species), Balitoridae (7 specimens of 1 speices), Bagridae (3 specimens of 1 species), Ambylcipitidae (1 specimen of 1 species), Siluridae (1 specimen of 1 species) and Cottidae (1 specimen of 1 species).

According to order level, Cypriniformes has 3 families, 34 species, 215 specimens followed by Perciformes (3 families, 4 species, 26 specimens), Siluriformes (3 families, 3 species, 5 specimens) and Scorpaeniformes (1 family, 1 species, 1 specimen).

We identified 17 species from 6 families of endemic fish in Korea. The endemic collection includes Acheilognathus signifier, A. yamatsutae, Coreoleuciscus splendidus, Gobiobotia brevibarba, G. macrocephala, Microphysogobio longidorsalis, M. yaluensis, Pseudopungtungia tenuicorpa, Rhodeus uyekii, Squalidus gracilis majimae, Zacco koreanus, Iksookimia koreensis, Koreocobitis rotundicaudata, Psudobagrus koreanus, Liobagrus andersoni, Coreoperca herzi and Odontobutis interrupta.

We found 5 endangered species designated by Ministry of Enviroment (Acheilognathus signifier, Gobiobotia 
Table 1. List of fish collection.

\begin{tabular}{|c|c|c|c|c|}
\hline Order & Family & Species & No. of specimens & Remark \\
\hline \multirow[t]{33}{*}{ Cypriniformes } & Cyprinidae & Abbottina rivularis & 4 & - \\
\hline & & Acheilognathus signifier & 1 & $\begin{array}{l}\text { Endemic, } \\
\text { Endangered }\end{array}$ \\
\hline & & Acheilognathus koreensis & 2 & - \\
\hline & & Acheilognathus lanceolatus & 5 & - \\
\hline & & Acheilognathus rhombeus & 7 & - \\
\hline & & Acheilognathus yamatsutae & 4 & Endemic \\
\hline & & Aphyocypris chinensis & 1 & - \\
\hline & & Carassius auratus & 9 & - \\
\hline & & Coreoleuciscus splendidus & 17 & Endemic \\
\hline & & Cyprinus carpio & 1 & - \\
\hline & & Gobiobotia brevibarba & 2 & $\begin{array}{l}\text { Endemic, } \\
\text { Endangered }\end{array}$ \\
\hline & & Gobiobotia macrocephalus & 1 & $\begin{array}{l}\text { Endemic, } \\
\text { Endangered }\end{array}$ \\
\hline & & Hemibarbus labeo & 5 & - \\
\hline & & Hemibarbus longirostris & 6 & Endemic \\
\hline & & Ladislavia taczanowski & 3 & - \\
\hline & & Microphysogobio longidorsalis & 3 & - \\
\hline & & Microphysogobio yaluensis & 8 & Endemic \\
\hline & & Pseudogobio esocinus & 12 & - \\
\hline & & Pseudorasbora parva & 6 & - \\
\hline & & Pseudopungtungia tenuicorpus & 2 & $\begin{array}{l}\text { Endemic, } \\
\text { Endangered }\end{array}$ \\
\hline & & Pungtungia herzi & 21 & - \\
\hline & & Rhodeus uyekii & 2 & Endemic \\
\hline & & Rhynchocypris oxycephalus & 13 & - \\
\hline & & Rhynchocypris steindachneri & 4 & - \\
\hline & & Sarcocheilichthys varieatus & 2 & - \\
\hline & & Squalidus gracilis & 3 & Endemic \\
\hline & & Zacco koreanus & 18 & Endemic \\
\hline & & Zacco platypus & 24 & - \\
\hline & Balitoridae & Orthrias nudus & 7 & - \\
\hline & Cobitidae & Iksookimia koreensis & 12 & Endemic \\
\hline & & Misgurnus anguillicaudatus & 6 & - \\
\hline & & Misgurnus mizolepis & 3 & - \\
\hline & & Koreocobitis rotundicaudata & 2 & Endemic \\
\hline \multirow[t]{3}{*}{ Siluriformes } & Bagridae & Pseudobagrus koreanus & 3 & Endemic \\
\hline & Ambylcipitadae & Liobagrus andersoni & 1 & Endemic \\
\hline & Siluridae & Silurus asotus & 1 & - \\
\hline Scorpaeniformes & Cottidae & Cottus koreanus & 1 & Endangered \\
\hline \multirow[t]{4}{*}{ Perciformes } & Centropomidae & Siniperca scherzeri & 2 & - \\
\hline & & Coreoperca herzi & 6 & Endemic \\
\hline & Odontobutidae & Odontobutis interrupta & 11 & Endemic \\
\hline & Gobiidae & Rhinogobius brunneus & 7 & - \\
\hline 4 orders & 10 families & 41 species & 248 & - \\
\hline
\end{tabular}

brevibarba, G. macrocephala, Pseudopungtungia tenuicorpa, Cottus koreanus).

In 2000, there was fish survey around Hantangang for Nature Environment Survey by the Ministry of Environment. The result of the previous survey shows 35 fish species along Hantangang (Chae and Yoon, 2000). The deference between two surveys might be cause by the number of field work. While field work was done two times for the survey in 2000, we did it four times.

\section{Amphibians and Retiles}

We collected 47 specimens ( 2 orders, 5 families, 10 species) of amphibians around Hantangan and adjacent 
Table 2. List of herpetofauna.

\begin{tabular}{|c|c|c|c|c|}
\hline Class & Order & Family & Species & No. of specimens \\
\hline \multirow[t]{10}{*}{ Amphibia } & Caudata & Hynobiidae & Hynobius leechii & 4 \\
\hline & & & Onychodactylus fischeri & 6 \\
\hline & Anura & Bufonidae & Bufo gargarizans & 3 \\
\hline & & & Bufo stejnegeri & 4 \\
\hline & & Bombinatoridae & Bombina orientalis & 5 \\
\hline & & Hylidae & Hyla japonica & 5 \\
\hline & & Ranidae & Pelophylax ngromaculatus & 12 \\
\hline & & & Glandirana emeljanovi & 2 \\
\hline & & & Rana dybowskii & 15 \\
\hline & & & Rana huanrenensis & 1 \\
\hline \multirow[t]{5}{*}{ Retilia } & Pholidota & Colubridae & Amphiesma vibakari & 1 \\
\hline & & & Elaphe dione & 5 \\
\hline & & & Elaphe rufodorsata & 2 \\
\hline & & & Rhabdophis tigrinus & 4 \\
\hline & & Viperidae & Gloydius ussuriensis & 7 \\
\hline 2 classes & 3 orders & 7 families & 15 species & 66 specimens \\
\hline
\end{tabular}

Table 3. List of birds along Hantangang.

\begin{tabular}{|c|c|c|c|c|}
\hline Order & Family & Species & No. of specimens & No. of individuals observed \\
\hline \multirow[t]{4}{*}{ Anseriformes } & Anatidae & Anser albifrons & - & 850 \\
\hline & & Anas crecca & - & 2 \\
\hline & & Anas platyrhynchos & - & 54 \\
\hline & & Anas poecilorhyncha & - & 68 \\
\hline \multirow[t]{2}{*}{ Falconiformes } & Accipitridae & Aegypius monachus & - & 4 \\
\hline & & Buteo buteo & - & 1 \\
\hline \multirow[t]{2}{*}{ Charadriiformes } & Charadriidae & Charadrius dubius & - & 1 \\
\hline & Scolopacidae & Actitis hypoleucos & - & 1 \\
\hline Columbiformes & Columbidae & Streptopelia orientalis & - & 1 \\
\hline Piciformes & Picidae & Dendrocopos kizuki & - & 1 \\
\hline \multirow[t]{15}{*}{ Passeriformes } & Motacillidae & Motacilla grandis & - & 1 \\
\hline & Pycnonotidae & Micorscelis amaurotis & 1 & 2 \\
\hline & Turdidea & Phoenicurus auroreus & 7 & 3 \\
\hline & Sylviidae & Urosphena squameiceps & 1 & \\
\hline & & Phylloscopus inornatus & 1 & \\
\hline & Aegithqalidae & Aegithalos caudatus & - & 6 \\
\hline & Paridae & Parus palustris & 3 & 5 \\
\hline & & Parus major & 7 & 2 \\
\hline & Emberizidae & Emberiza tristrami & 1 & \\
\hline & & Emberiza rustica & 1 & 8 \\
\hline & & Emberiza elegans & 27 & 1 \\
\hline & & Emberiza spodocephala & 2 & \\
\hline & Corvidae & Garrulus glandarius & - & 2 \\
\hline & & Pica pica & - & 28 \\
\hline & & Corvus macrorhynchos & - & 4 \\
\hline 6 order & 14 families & 25 species & 51 specimens & \\
\hline
\end{tabular}

area including montane creeks (Table 2). We also caught, 19 specimens ( 1 order, 2 families, 5 species) of reptiles.
In the amphibian collection, we identified 4 individuals of Korean salamander Hynobius leechii, 6 Long-tailed 
Table 4. List of mammals along Hantangang.

\begin{tabular}{|c|c|c|c|c|}
\hline Order & Family & Species & No. of specimen & Remark \\
\hline Lagomorpha & Leporidae & Lepus coreanus & - & Field sign \\
\hline \multirow[t]{2}{*}{ Soricomorpha } & Soricidae & Crocidura lasiura & 9 & \\
\hline & Talpidae & Mogera wogura & - & Field sign \\
\hline \multirow[t]{3}{*}{ Carnivora } & Felidae & Prionailurus bengalensis & - & Field sign, Endangered II \\
\hline & Canidae & Nyctereutes procyonoides & 1 & \\
\hline & Mustelidae & $\begin{array}{l}\text { Lutra lutra } \\
\text { Mustela sibirica }\end{array}$ & $\begin{array}{l}- \\
-\end{array}$ & $\begin{array}{l}\text { Field sign, Endangered I } \\
\text { Direct sight }\end{array}$ \\
\hline \multirow[t]{2}{*}{ Artiodactyla } & Suidae & Sus scrofa & - & Field sign \\
\hline & Cervidae & Hydropotes inermis & 2 & \\
\hline \multirow[t]{2}{*}{ Rodentia } & Sciuridae & $\begin{array}{l}\text { Sciurus vulgaris } \\
\text { Tamias sibiricus }\end{array}$ & $\begin{array}{c}- \\
1\end{array}$ & Direct sight \\
\hline & Muridae & $\begin{array}{l}\text { Apodemus agrarius } \\
\text { Mus mиsculus }\end{array}$ & $\begin{array}{r}73 \\
4\end{array}$ & \\
\hline 5 orders & 10 families & 13 species & 90 & \\
\hline
\end{tabular}

Clawed Salamander Onychodactylus fischeri, 3 Asiatic toad Bufo gargarizans, 4 Korean water toad Bufo stejnegeri, 5 Oriental Fire-bellied Toad Bombina orientalis, 5 Japanese tree frog Hyla japonica, 12 black-spotted frog Pelophylax nigromaculatus, 2 Imienpo Station frog Glandirana emeljanovi, 15 Dybowski's frog Rana dybowskii and 1 Huanren frog Rana huanrenensis.

For the reptile survey, we collected 1 Asian keelback Amphiesma vibakari, 5 Dione Ratsnake Elaphe dione, 2 Red-backed Ratsnake Elaphe rufodorsata, 4 tiger keelback Rhabdophis tigrinus and 7 Ussuri mamushi Gloydius ussuriensis.

This amphians result shows much abundant than previous survey in 2000. The amphibian survey in 2000 shows that 5 species of amphibians were identified around the research site (Han et al., 2000). Unlike amphibian, the number of reptile found is same in both surveys. This result might be caused by extensive field survey focusing on riparian habitats. While this survey was done along riparian habitats, previous national survey was focused on mountane habitats.

\section{Birds}

We collected 51 bird specimens ( 1 order, 5 families, 10 species) and identified 25 species of birds around Hantangang (Table 3). Yellow-throated bunting Emberiza elegans $(n=27)$ was the most abundant collected species followed by Daurian redstart Phoenicurus auroreus $(n=7)$, great tit Parus major $(n=7)$, marsh tit Parus palustris ( $n$ $=3)$, black-faced bunting Emberiza spodocephala $(n=2)$ and 1 specimen each of brown eared bulbul Micorscelis amaurotis, yellolw-browed warbler Phylloscopus inornatus, rustic bunting Emberiza rustica, Asian stubtail
Urosphena squameiceps and Tristram's bunting Emberiza tristrami.

Through field observations, we've seen 850 of whitefronted geese Anser albifrons, followed by spot-billed duck Anas poecilorhyncha (68) and mallard Anas platyrhynchos (54).

For bird trapping, mist net is one the most general methods but it is limited for lower flying birds and species prefer shrub habitats (Bub, 1991). It's necessary to use various methods for identifying more bird species.

\section{Mammals}

Along the riparian habitat and river banks of Hantangang, 4 species of small mammals were caught; the Ussuri white-toothed shrew Crocidura lasiura, Siberian chipmunk Tamias sibiricus, striped field mouse Apodemus agrarius and house mouse Mus musculus. We also collected dead bodies of raccoon dog (Nyctereutes procyonoides) and water deer (Hydropotes inermis) around Hantangang (Table 4). Finally, we identified lesser Japanese mole Mogera wogura, Eurasian otter Lutra lutra, Siberian weasel Mustela sibirica, leopard cat Prionailurus bengalensis, wild boar Sus scrofa, red squirrel Sciurus vulgaris and Korean hare Lepus coreanus by field sign and direct observation. We listed a total of 13 species from 10 families (Table 4).

During spring season trapping among the kill traps, there has been 5 loss of mouse after capture. It might be caused by predator or scavensor species. Also, there has been some damage on the skin of sample caught by kill traps. For getting nice collection, we recommend a live trap protected by outside. 


\section{ACKNOWLedgements}

Dr. Seon-Man Kweon, Seung-Ki Lee, Dr. Sang-Cheol Lee and Hong Seomun helped this collection. We thank Jonathan J. Derbridge (School of Natural Resources, University of Arizona) for helpful conversation about this manuscript. 3 anonymous reviewers provided valuable comments that significantly improved the manuscript. This survey was funded by NIBR Research fund.

\section{REFERENCES}

Bub, H. 1991. Bird Trapping and Bird Banding. Cornell University Press, New York, 330 pp.

Chae, B.S. and H.N. Yoon. 2000. Freshwater fish in Yeoncheon and Pocheon: Second Natural Environment Survey, Ministry of Environemt, Gwacheon (in Korean).

Han, S.Y., D.H. Oh, J.W. Ha, B.J. Jo and S.H. Han. 2000. Mammals, Amphibians and Retititles in Yeoncheon and Cheorwon: Second Natural Environment Survey, Ministry of Environemt, Gwacheon (in Korean).

Johnson, K.M. 2001. Hantaviruses: History and Overview. In: Hantaviruses: current topics in microbiology and immunology (C.S. Schmaljohn and S.T. Nichol, eds.), Springer, New York, pp. 1-11.

Jones, C., W.J. McShea, M.J. Conroy and T.H. Kunz. 1996. Capturing mammals. In: Measuring and /monitoring Biological Diversity: Standard Methods for Mammals (Wil- son, D.E., F.R. Cole, J.D. Nichols, R. Rudran and M.S. Forster eds.), Smithsonian Institute Press, Washington, pp. 115-155.

Kim, I.S., M.K. Oh and K. Hosoya. 2005. A new species of cyprinid fish, Zacco koreanus with redescription of $Z$. temminckii (Cyprinidae) from Korea. Korean Journal of Ichthyology 17:1-7.

McCully, P. 2001. Silenced Rivers: The Ecology and Politics of Large Dams. Zed books, London, 416 pp.

National Institute of Biological Resources. 2007. Policy Establishment of Biological Resources in National Institute of Biological Resources. NIBR, Incheon, 453p (in Korean).

Nelson, J.S. 2006. Fishes of the world ( $4^{\text {th }}$ edit.). John Wiley \& Sons, New Jersey, $601 \mathrm{pp}$.

Tucker, G. 2005. Biodiversity Evaluation Methods. In: Handbook of Biodiversity Methods. In: D. Hill, M. Fasham, G. Tucker, M. Shewry and P. Shaw (eds.), Cambridge press, NewYork, pp. 65-101.

Van Dyke, F. 2008. Conservation Biology: Foundation, Concepts, Applications. Springer, Dordrecht, 477 pp.

Won, C.M. and K.G. Smith. 1999. History and current status of mammals of the Korean Peninsula. Mammal Review 29:3-33.

Won, J.G., M.W. Lee, M.S. Jin, M.J. Choi and B.H. Jeong. 2010. Hantangang Geology Exporering. Jiseongsa, Seoul, 232 pp (in Korean).

Submitted: January 25, 2012, Accepted: February 9, 2012 
Appendix 1. Collection list of fish.

\begin{tabular}{|c|c|c|c|c|c|c|}
\hline Collection no. & Family & Genus & Species & Korean name & Date & Collecter \\
\hline NIBR-P2470 & COBITIDAE & Iksookimia & koreensis & 참종개 & 2008-04-23 & BJ Kim, SM Kweon \\
\hline NIBR-P2471 & BALITORIDAE & Orthrias & nudus & 대륙종개 & 2008-04-23 & BJ Kim, SM Kweon \\
\hline NIBR-P2472 & CYPRINIDAE & Zacco & koreanus & 참갈겨니 & $2008-04-23$ & BJ Kim, SM Kweon \\
\hline NIBR-P2473 & CYPRINIDAE & Rhynchocypris & oxycephalus & 버들치 & 2008-04-23 & BJ Kim, SM Kweon \\
\hline NIBR-P2474 & CYPRINIDAE & Rhynchocypris & steindachneri & 버들개 & 2008-04-23 & BJ Kim, SM Kweon \\
\hline NIBR-P2475 & COBITIDAE & Misgurnus & anguillicaudatus & 미꾸리 & 2008-04-23 & BJ Kim, SM Kweon \\
\hline NIBR-P2476 & CYPRINIDAE & Microphysogobio & longidorsalis & 배가사리 & 2008-04-23 & BJ Kim, SM Kweon \\
\hline NIBR-P2477 & CYPRINIDAE & Pungtungia & herzi & 돌고기 & 2008-04-23 & BJ Kim, SM Kweon \\
\hline NIBR-P2478 & CYPRINIDAE & Microphysogobio & yaluensis & 돌마자 & 2008-04-23 & BJ Kim, SM Kweon \\
\hline NIBR-P2479 & CYPRINIDAE & Microphysogobio & yaluensis & 돌마자 & 2008-04-23 & BJ Kim, SM Kweon \\
\hline NIBR-P2480 & CYPRINIDAE & Zacco & platypus & 피라미 & 2008-04-23 & BJ Kim, SM Kweon \\
\hline NIBR-P2481 & CYPRINIDAE & Pseudogobio & esocinus & 모래무지 & 2008-04-23 & BJ Kim, SM Kweon \\
\hline NIBR-P2482 & SILURIDAE & Silurus & asotus & 메기 & 2008-04-23 & BJ Kim, SM Kweon \\
\hline NIBR-P2483 & GOBIIDAE & Rhinogobius & brunneus & 밀어 & 2008-04-23 & BJ Kim, SM Kweon \\
\hline NIBR-P2484 & ODONTOBUTIDAE & Odontobutis & interrupta & 얼록동사리 & 2008-04-23 & BJ Kim, SM Kweon \\
\hline NIBR-P2485 & AMBLYCIPITIDAE & Liobagrus & andersoni & 퉁가리 & 2008-04-23 & BJ Kim, SM Kweon \\
\hline NIBR-P2486 & CYPRINIDAE & Acheilognathus & koreensis & 칼납자루 & 2008-04-23 & BJ Kim, SM Kweon \\
\hline NIBR-P2487 & CYPRINIDAE & Coreoleuciscus & splendidus & 쉬리 & 2008-04-23 & BJ Kim, SM Kweon \\
\hline NIBR-P2488 & CENTROPOMIDAE & Siniperca & scherzeri & 쏘가리 & 2008-04-23 & BJ Kim, SM Kweon \\
\hline NIBR-P2489 & CYPRINIDAE & Pseudogobio & esocinus & 모래무지 & 2008-04-23 & BJ Kim, SM Kweon \\
\hline NIBR-P2490 & CYPRINIDAE & Pungtungia & herzi & 돌고기 & 2008-04-23 & BJ Kim, SM Kweon \\
\hline NIBR-P2491 & CYPRINIDAE & Zacco & platypus & 피라미 & 2008-04-23 & BJ Kim, SM Kweon \\
\hline NIBR-P2492 & CYPRINIDAE & Sarcocheilichthys & varieatus & 참중고기 & 2008-04-23 & BJ Kim, SM Kweon \\
\hline NIBR-P2493 & COBITIDAE & Misgurnus & anguillicaudatus & 미꾸리 & 2008-04-24 & BJ Kim \\
\hline NIBR-P2494 & CYPRINIDAE & Coreoleuciscus & splendidus & 쉬리 & 2008-04-24 & BJ Kim \\
\hline NIBR-P2495 & CYPRINIDAE & Gobiobotia & brevibarba & 돌상어 & 2008-04-24 & BJ Kim \\
\hline NIBR-P2496 & CYPRINIDAE & Hemibarbus & labeo & 누치 & 2008-04-24 & BJ Kim \\
\hline NIBR-P2497 & CYPRINIDAE & Carassius & auratus & 붕어 & 2008-04-24 & BJ Kim \\
\hline NIBR-P2498 & CYPRINIDAE & Pungtungia & herzi & 돌고기 & 2008-04-24 & BJ Kim \\
\hline NIBR-P2499 & COBITIDAE & Misgurnus & anguillicaudatus & 미꾸리 & 2008-04-24 & BJ Kim \\
\hline NIBR-P2500 & BALITORIDAE & Orthrias & nudus & 대륙종개 & 2008-04-24 & BJ Kim \\
\hline NIBR-P2501 & CYPRINIDAE & Zacco & platypus & 피라미 & 2008-04-24 & BJ Kim \\
\hline NIBR-P2502 & COBITIDAE & Iksookimia & koreensis & 참종개 & 2008-04-24 & BJ Kim \\
\hline NIBR-P2503 & CYPRINIDAE & Abbottina & rivularis & 버들매치 & 2008-04-24 & BJ Kim \\
\hline NIBR-P2504 & CYPRINIDAE & Pseudorasbora & parva & 참붕어 & 2008-04-24 & BJ Kim \\
\hline NIBR-P2505 & CYPRINIDAE & Acheilognathus & koreensis & 칼납자루 & 2008-04-24 & BJ Kim \\
\hline NIBR-P2506 & CYPRINIDAE & Ladislavia & taczanowski & 새미 & 2008-04-24 & BJ Kim \\
\hline NIBR-P2507 & CYPRINIDAE & Zacco & koreanus & 참갈겨니 & 2008-04-24 & BJ Kim \\
\hline NIBR-P2508 & CYPRINIDAE & Rhynchocypris & oxycephalus & 버들치 & 2008-04-24 & BJ Kim \\
\hline NIBR-P2509 & COBITIDAE & Iksookimia & koreensis & 참종개 & 2008-04-24 & BJ Kim \\
\hline NIBR-P2510 & CYPRINIDAE & Pseudorasbora & parva & 참붕어 & 2008-04-24 & BJ Kim \\
\hline NIBR-P2511 & BALITORIDAE & Orthrias & nudus & 대륙종개 & 2008-04-24 & BJ Kim \\
\hline NIBR-P2512 & CYPRINIDAE & Rhynchocypris & oxycephalus & 버들치 & 2008-04-24 & BJ Kim \\
\hline NIBR-P2513 & CYPRINIDAE & Ladislavia & taczanowski & 새미 & 2008-04-24 & BJ Kim \\
\hline NIBR-P2514 & CYPRINIDAE & Zacco & platypus & 피라미 & 2008-04-24 & BJ Kim \\
\hline NIBR-P2515 & CYPRINIDAE & Zacco & koreanus & 참갈겨니 & $2008-04-25$ & BJ Kim \\
\hline NIBR-P2516 & CYPRINIDAE & Rhynchocypris & steindachneri & 버들개 & 2008-04-25 & BJ Kim \\
\hline NIBR-P2517 & CYPRINIDAE & Pseudorasbora & parva & 참붕어 & $2008-04-25$ & BJ Kim \\
\hline NIBR-P2518 & CYPRINIDAE & Zассо & platypus & 피라미 & $2008-04-25$ & BJ Kim \\
\hline NIBR-P2519 & CYPRINIDAE & Carassius & auratus & 붕어 & 2008-04-25 & BJ Kim \\
\hline NIBR-P2520 & BALITORIDAE & Orthrias & nudus & 대륙종개 & 2008-04-25 & BJ Kim \\
\hline NIBR-P2521 & COBITIDAE & Misgurnus & anguillicaudatus & 미꾸리 & $2008-04-25$ & BJ Kim \\
\hline NIBR-P2522 & COBITIDAE & Misgurnus & mizolepis & 미꾸라지 & 2008-04-25 & BJ Kim \\
\hline NIBR-P2523 & CYPRINIDAE & Rhynchocypris & oxycephalus & 버들치 & 2008-04-25 & BJ Kim \\
\hline NIBR-P2805 & CYPRINIDAE & Pungtungia & herzi & 돌고기 & $2008-07-23$ & BJ Kim, SM Kweon \\
\hline NIBR-P2806 & CYPRINIDAE & Zacco & platypus & 피라미 & $2008-07-23$ & BJ Kim, SM Kweon \\
\hline NIBR-P2807 & CYPRINIDAE & Abbottina & rivularis & 버들매치 & $2008-07-23$ & BJ Kim, SM Kweon \\
\hline NIBR-P2808 & CYPRINIDAE & Microphysogobio & yaluensis & 돌마자 & $2008-07-23$ & BJ Kim, SM Kweon \\
\hline NIBR-P2809 & CYPRINIDAE & Pseudogobio & esocinus & 모래무지 & 2008-07-23 & BJ Kim, SM Kweon \\
\hline NIBR-P2810 & CYPRINIDAE & Hemibarbus & longirostris & 참마자 & $2008-07-23$ & BJ Kim, SM Kweon \\
\hline NIBR-P2811 & CYPRINIDAE & Carassius & auratus & 붕어 & $2008-07-23$ & BJ Kim, SM Kweon \\
\hline
\end{tabular}


Appendix 1. Continued.

\begin{tabular}{|c|c|c|c|c|c|c|}
\hline Collection no. & Family & Genus & Species & Korean name & Date & Collecter \\
\hline NIBR-P2812 & CYPRINIDAE & Rhynchocypris & oxycephalus & 버들치 & $2008-07-23$ & BJ Kim, SM Kweon \\
\hline NIBR-P2813 & CYPRINIDAE & Squalidus & gracilis & 긴몰개 & $2008-07-23$ & BJ Kim, SM Kweon \\
\hline NIBR-P2814 & BALITORIDAE & Orthrias & nudus & 대륙종개 & $2008-07-23$ & BJ Kim, SM Kweon \\
\hline NIBR-P2815 & ODONTOBUTIDAE & Odontobutis & interrupta & 얼록동사리 & $2008-07-23$ & BJ Kim, SM Kweon \\
\hline NIBR-P2816 & CYPRINIDAE & Zacco & koreanus & 참갈겨니 & $2008-07-23$ & BJ Kim, SM Kweon \\
\hline NIBR-P2817 & CYPRINIDAE & Zacco & platypus & 피라미 & $2008-07-23$ & BJ Kim, SM Kweon \\
\hline NIBR-P2818 & CYPRINIDAE & Zacco & koreanus & 참갈겨니 & $2008-07-23$ & BJ Kim, SM Kweon \\
\hline NIBR-P2819 & CYPRINIDAE & Hemibarbus & labeo & 누치 & $2008-07-23$ & BJ Kim, SM Kweon \\
\hline NIBR-P2820 & CYPRINIDAE & Acheilognathus & yamatsutae & 줄납자루 & $2008-07-23$ & BJ Kim, SM Kweon \\
\hline NIBR-P2821 & CYPRINIDAE & Coreoleuciscus & splendidus & 쉬리 & 2008-07-23 & BJ Kim, SM Kweon \\
\hline NIBR-P2822 & CYPRINIDAE & Pseudogobio & esocinus & 모래무지 & $2008-07-23$ & BJ Kim, SM Kweon \\
\hline NIBR-P2823 & CYPRINIDAE & Pungtungia & herzi & 돌고기 & $2008-07-23$ & BJ Kim, SM Kweon \\
\hline NIBR-P2824 & CYPRINIDAE & Pungtungia & herzi & 가는돌고기 & 2008-07-23 & BJ Kim, SM Kweon \\
\hline NIBR-P2825 & CENTROPOMIDAE & Siniperca & scherzeri & 쏘가리 & $2008-07-23$ & BJ Kim, SM Kweon \\
\hline NIBR-P2826 & CYPRINIDAE & Microphysogobio & longidorsalis & 배가사리 & $2008-07-23$ & BJ Kim, SM Kweon \\
\hline NIBR-P2827 & CYPRINIDAE & Microphysogobio & yaluensis & 돌마자 & $2008-07-23$ & BJ Kim, SM Kweon \\
\hline NIBR-P2828 & CYPRINIDAE & Acheilognathus & rhombeus & 납지리 & $2008-07-23$ & BJ Kim, SM Kweon \\
\hline NIBR-P2829 & CYPRINIDAE & Acheilognathus & & 납자루속 & $2008-07-23$ & BJ Kim, SM Kweon \\
\hline NIBR-P3192 & CYPRINIDAE & Zacco & platypus & 피라미 & 2008-07-24 & $\begin{array}{l}\text { BJ Kim, SM Kweon, } \\
\text { SK Lee }\end{array}$ \\
\hline NIBR-P3193 & CYPRINIDAE & Coreoleuciscus & splendidus & 쉬리 & 2008-07-24 & $\begin{array}{l}\text { BJ Kim, SM Kweon, } \\
\text { SK Lee }\end{array}$ \\
\hline NIBR-P3194 & CYPRINIDAE & Zacco & platypus & 피라미 & 2008-07-24 & $\begin{array}{l}\text { BJ Kim, SM Kweon, } \\
\text { SK Lee }\end{array}$ \\
\hline NIBR-P3344 & CYPRINIDAE & Pseudogobio & esocinus & 모래무지 & 2008-10-06 & BJ Kim \\
\hline NIBR-P3345 & CYPRINIDAE & Zacco & platypus & 피라미 & 2008-10-06 & BJ Kim \\
\hline NIBR-P3346 & CYPRINIDAE & Zacco & koreanus & 참갈겨니 & 2008-10-06 & BJ Kim \\
\hline NIBR-P3347 & GOBIIDAE & Rhinogobius & brunneus & 밀어 & 2008-10-06 & BJ Kim \\
\hline NIBR-P3348 & CYPRINIDAE & Squalidus & gracilis & 긴몰개 & 2008-10-06 & BJ Kim \\
\hline NIBR-P3349 & COBITIDAE & Iksookimia & koreensis & 참종개 & 2008-10-06 & BJ Kim \\
\hline NIBR-P3350 & CYPRINIDAE & Rhynchocypris & oxycephalus & 버들치 & 2008-10-06 & BJ Kim \\
\hline NIBR-P3351 & CYPRINIDAE & Microphysogobio & yaluensis & 돌마자 & 2008-10-06 & BJ Kim \\
\hline NIBR-P3352 & CYPRINIDAE & Pungtungia & herzi & 돌고기 & 2008-10-06 & BJ Kim \\
\hline NIBR-P3353 & CYPRINIDAE & Coreoleuciscus & splendidus & 쉬리 & 2008-10-06 & BJ Kim \\
\hline NIBR-P3354 & CYPRINIDAE & Zacco & platypus & 피라미 & 2008-10-06 & BJ Kim \\
\hline NIBR-P3355 & CYPRINIDAE & Hemibarbus & longirostris & 참마자 & 2008-10-06 & BJ Kim \\
\hline NIBR-P3356 & BALITORIDAE & Orthrias & nudus & 대륙종개 & 2008-10-06 & BJ Kim \\
\hline NIBR-P3357 & COBITIDAE & Iksookimia & koreensis & 참종개 & 2008-10-06 & BJ Kim \\
\hline NIBR-P3358 & CYPRINIDAE & Coreoleuciscus & splendidus & 쉬리 & 2008-10-06 & BJ Kim \\
\hline NIBR-P3359 & CYPRINIDAE & Microphysogobio & yaluensis & 돌마자 & 2008-10-06 & BJ Kim \\
\hline NIBR-P3360 & CYPRINIDAE & Pseudorasbora & parva & 참붕어 & 2008-10-06 & BJ Kim \\
\hline NIBR-P3361 & CYPRINIDAE & Zacco & koreanus & 참갈겨니 & 2008-10-06 & BJ Kim \\
\hline NIBR-P3362 & BALITORIDAE & Orthrias & nudus & 대륙종개 & 2008-10-06 & BJ Kim \\
\hline NIBR-P3363 & CYPRINIDAE & Pseudogobio & esocinus & 모래무지 & 2008-10-06 & BJ Kim \\
\hline NIBR-P3364 & CYPRINIDAE & Coreoleuciscus & splendidus & 쉬리 & 2008-10-06 & BJ Kim \\
\hline NIBR-P3365 & CYPRINIDAE & Hemibarbus & longirostris & 참마자 & 2008-10-06 & BJ Kim \\
\hline NIBR-P3366 & CYPRINIDAE & Microphysogobio & yaluensis & 돌마자 & 2008-10-06 & BJ Kim \\
\hline NIBR-P3367 & GOBIIDAE & Rhinogobius & brunneus & 밀어 & 2008-10-06 & BJ Kim \\
\hline NIBR-P3368 & CYPRINIDAE & Pungtungia & herzi & 돌고기 & 2008-10-06 & BJ Kim \\
\hline NIBR-P3369 & CYPRINIDAE & Zacco & platypus & 피라미 & 2008-10-06 & BJ Kim \\
\hline NIBR-P3370 & CYPRINIDAE & Hemibarbus & labeo & 누치 & 2008-10-07 & BJ Kim \\
\hline NIBR-P3371 & CYPRINIDAE & Pungtungia & herzi & 돌고기 & 2008-10-07 & BJ Kim \\
\hline NIBR-P3372 & CYPRINIDAE & Zacco & platypus & 피라미 & 2008-10-07 & BJ Kim \\
\hline NIBR-P3373 & GOBIIDAE & Rhinogobius & brunneus & 밀어 & 2008-10-07 & BJ Kim \\
\hline NIBR-P3374 & CYPRINIDAE & Coreoleuciscus & splendidus & 쉬리 & 2008-10-07 & BJ Kim \\
\hline NIBR-P3375 & CYPRINIDAE & Gobiobotia & macrocephalus & 꾸꾸리 & 2008-10-07 & BJ Kim \\
\hline NIBR-P3376 & CYPRINIDAE & Zacco & platypus & 피라미 & 2008-10-07 & BJ Kim \\
\hline NIBR-P3377 & CYPRINIDAE & Pungtungia & herzi & 돌고기 & 2008-10-07 & BJ Kim \\
\hline NIBR-P3378 & CYPRINIDAE & Zacco & koreanus & 참갈겨니 & 2008-10-07 & BJ Kim \\
\hline NIBR-P3379 & CYPRINIDAE & Acheilognathus & yamatsutae & 줄납자루 & 2008-10-07 & BJ Kim \\
\hline NIBR-P3380 & COBITIDAE & Koreocobitis & rotundicaudata & 새코미꾸리 & 2008-10-07 & BJ Kim \\
\hline
\end{tabular}


Appendix 1. Continued.

\begin{tabular}{|c|c|c|c|c|c|c|}
\hline Collection no. & Family & Genus & Species & Korean name & Date & Collecter \\
\hline NIBR-P3381 & BAGRIDAE & Pseudobagrus & koreanus & 눈동자개 & 2008-10-07 & BJ Kim \\
\hline NIBR-P3382 & CYPRINIDAE & Pseudogobio & esocinus & 모래무지 & 2008-10-07 & BJ Kim \\
\hline NIBR-P3383 & CYPRINIDAE & Zacco & platypus & 피라미 & 2008-10-07 & BJ Kim \\
\hline NIBR-P3384 & ODONTOBUTIDAE & Odontobutis & interrupta & 얼록동사리 & 2008-10-07 & BJ Kim \\
\hline NIBR-P3385 & CYPRINIDAE & Rhynchocypris & oxycephalus & 버들치 & 2008-10-07 & BJ Kim \\
\hline NIBR-P3386 & GOBIIDAE & Rhinogobius & brunneus & 밀어 & 2008-10-07 & BJ Kim \\
\hline NIBR-P3387 & CYPRINIDAE & Zacco & platypus & 피라미 & $2008-10-07$ & BJ Kim \\
\hline NIBR-P3388 & CYPRINIDAE & Zacco & koreanus & 참갈겨니 & $2008-10-07$ & BJ Kim \\
\hline NIBR-P3389 & CYPRINIDAE & Cyprinus & carpio & 잉어 & 2008-10-07 & BJ Kim \\
\hline NIBR-P3390 & CYPRINIDAE & Carassius & auratus & 붕어 & 2008-10-07 & BJ Kim \\
\hline NIBR-P3391 & CYPRINIDAE & Pseudogobio & esocinus & 모래무지 & 2008-10-07 & BJ Kim \\
\hline NIBR-P3392 & CYPRINIDAE & Microphysogobio & longidorsalis & 배가사리 & 2008-10-07 & BJ Kim \\
\hline NIBR-P3393 & SILURIDAE & Silurus & asotus & 메기 & 2008-10-07 & BJ Kim \\
\hline NIBR-P3394 & CYPRINIDAE & Hemibarbus & labeo & 누치 & 2008-10-07 & BJ Kim \\
\hline NIBR-P3395 & ODONTOBUTIDAE & Odontobutis & interrupta & 얼록동사리 & 2008-10-07 & BJ Kim \\
\hline NIBR-P3396 & CYPRINIDAE & Microphysogobio & yaluensis & 돌마자 & 2008-10-07 & BJ Kim \\
\hline NIBR-P3397 & CYPRINIDAE & Acheilognathus & rhombeus & 납지리 & 2008-10-07 & BJ Kim \\
\hline NIBR-P3398 & CYPRINIDAE & Pungtungia & herzi & 돌고기 & $2008-10-07$ & BJ Kim \\
\hline NIBR-P3399 & GOBIIDAE & Rhinogobius & brunneus & 밀어 & 2008-10-07 & BJ Kim \\
\hline NIBR-P3400 & CYPRINIDAE & Hemibarbus & longirostris & 참마자 & 2008-10-07 & BJ Kim \\
\hline NIBR-P3401 & CYPRINIDAE & Rhynchocypris & steindachneri & 버들개 & 2008-10-07 & BJ Kim \\
\hline NIBR-P3402 & CYPRINIDAE & Rhynchocypris & oxycephalus & 버들치 & 2008-10-07 & BJ Kim \\
\hline NIBR-P3403 & CENTROPOMIDAE & Coreoperca & herzi & 꺽지 & $2008-10-07$ & BJ Kim \\
\hline NIBR-P3404 & COBITIDAE & Koreocobitis & rotundicaudata & 새코미꾸리 & 2008-10-08 & BJ Kim \\
\hline NIBR-P3405 & CYPRINIDAE & Pseudogobio & esocinus & 모래무지 & 2008-10-08 & BJ Kim \\
\hline NIBR-P3406 & CYPRINIDAE & Pungtungia & herzi & 돌고기 & 2008-10-08 & BJ Kim \\
\hline NIBR-P3407 & CYPRINIDAE & Coreoleuciscus & splendidus & 쉬리 & $2008-10-08$ & BJ Kim \\
\hline NIBR-P3408 & BAGRIDAE & Pseudobagrus & koreanus & 눈동자개 & 2008-10-08 & BJ Kim \\
\hline NIBR-P3409 & ODONTOBUTIDAE & Odontobutis & interrupta & 얼록동사리 & 2008-10-08 & BJ Kim \\
\hline NIBR-P3410 & CYPRINIDAE & Acheilognathus & rhombeus & 납지리 & 2008-10-08 & BJ Kim \\
\hline NIBR-P3411 & COBITIDAE & Iksookimia & koreensis & 참종개 & 2008-10-08 & BJ Kim \\
\hline NIBR-P3412 & CYPRINIDAE & Zacco & koreanus & 참갈겨니 & $2008-10-08$ & BJ Kim \\
\hline NIBR-P3413 & CYPRINIDAE & Zacco & platypus & 피라미 & $2008-10-08$ & BJ Kim \\
\hline NIBR-P3414 & CYPRINIDAE & Pseudogobio & esocinus & 모래무지 & 2008-10-08 & BJ Kim \\
\hline NIBR-P3415 & ODONTOBUTIDAE & Odontobutis & interrupta & 얼록동사리 & $2008-10-08$ & BJ Kim \\
\hline NIBR-P3416 & CYPRINIDAE & Acheilognathus & rhombeus & 납지리 & 2008-10-08 & BJ Kim \\
\hline NIBR-P3417 & CYPRINIDAE & Coreoleuciscus & splendidus & 쉬리 & 2008-10-08 & BJ Kim \\
\hline NIBR-P3418 & CYPRINIDAE & Zacco & platypus & 피라미 & 2008-10-08 & BJ Kim \\
\hline NIBR-P3419 & CYPRINIDAE & Zacco & koreanus & 참갈겨니 & 2008-10-08 & BJ Kim \\
\hline NIBR-P3420 & COBITIDAE & Iksookimia & koreensis & 참종개 & 2008-10-08 & BJ Kim \\
\hline NIBR-P3421 & CYPRINIDAE & Pungtungia & herzi & 돌고기 & $2008-10-08$ & BJ Kim \\
\hline NIBR-P3422 & CYPRINIDAE & Squalidus & gracilis & 긴몰개 & $2008-10-08$ & BJ Kim \\
\hline NIBR-P3423 & CYPRINIDAE & Rhodeus & uyekii & 각시붕어 & 2008-10-08 & BJ Kim \\
\hline NIBR-P3424 & CYPRINIDAE & Acheilognathus & yamatsutae & 줄납자루 & $2008-10-08$ & BJ Kim \\
\hline NIBR-P3425 & CYPRINIDAE & Acheilognathus & lanceolatus & 납자루 & 2008-10-08 & BJ Kim \\
\hline NIBR-P3426 & CYPRINIDAE & Zacco & koreanus & 참갈겨니 & 2008-10-08 & BJ Kim \\
\hline NIBR-P3427 & CYPRINIDAE & Coreoleuciscus & splendidus & 쉬리 & 2008-10-08 & BJ Kim \\
\hline NIBR-P3428 & CENTROPOMIDAE & Coreoperca & herzi & 꺽지 & 2008-10-08 & BJ Kim \\
\hline NIBR-P3429 & COBITIDAE & Iksookimia & koreensis & 참종개 & 2008-10-08 & BJ Kim \\
\hline NIBR-P3430 & ODONTOBUTIDAE & Odontobutis & interrupta & 얼록동사리 & $2008-10-08$ & BJ Kim \\
\hline NIBR-P3431 & CYPRINIDAE & Pungtungia & herzi & 돌고기 & 2008-10-08 & BJ Kim \\
\hline NIBR-P3432 & CYPRINIDAE & Pungtungia & herzi & 돌고기 & 2008-10-08 & BJ Kim \\
\hline NIBR-P3433 & CYPRINIDAE & Rhynchocypris & oxycephalus & 버들치 & $2008-10-08$ & BJ Kim \\
\hline NIBR-P3434 & ODONTOBUTIDAE & Odontobutis & interrupta & 얼록동사리 & $2008-10-08$ & BJ Kim \\
\hline NIBR-P3435 & CYPRINIDAE & Coreoleuciscus & splendidus & 쉬리 & 2008-10-08 & BJ Kim \\
\hline NIBR-P3436 & CENTROPOMIDAE & Coreoperca & herzi & 꺽지 & 2008-10-08 & BJ Kim \\
\hline NIBR-P3437 & COBITIDAE & Iksookimia & koreensis & 참종개 & $2008-10-08$ & BJ Kim \\
\hline NIBR-P3438 & COTTIDAE & Cottus & koreanus & 둑중개 & 2008-10-08 & BJ Kim \\
\hline NIBR-P3439 & BALITORIDAE & Orthrias & nudus & 대륙종개 & 2008-10-09 & BJ Kim \\
\hline NIBR-P3440 & CYPRINIDAE & Coreoleuciscus & splendidus & 쉬리 & 2008-10-09 & BJ Kim \\
\hline NIBR-P3441 & CYPRINIDAE & Pungtungia & herzi & 돌고기 & 2008-10-09 & BJ Kim \\
\hline
\end{tabular}


Appendix 1. Continued.

\begin{tabular}{|c|c|c|c|c|c|c|}
\hline Collection no. & Family & Genus & Species & Korean name & Date & Collecter \\
\hline NIBR-P3442 & CYPRINIDAE & Zacco & koreanus & 참갈겨니 & 2008-10-09 & BJ Kim \\
\hline NIBR-P3443 & COBITIDAE & Iksookimia & koreensis & 참종개 & 2008-10-09 & BJ Kim \\
\hline NIBR-P3444 & COBITIDAE & Misgurnus & anguillicaudatus & 미꾸리 & 2008-10-09 & BJ Kim \\
\hline NIBR-P3445 & CYPRINIDAE & Acheilognathus & lanceolatus & 납자루 & 2008-10-09 & BJ Kim \\
\hline NIBR-P3446 & CYPRINIDAE & Rhynchocypris & oxycephalus & 버들치 & 2008-10-09 & BJ Kim \\
\hline NIBR-P3447 & CYPRINIDAE & Rhodeus & uyekii & 각시붕어 & 2008-10-09 & BJ Kim \\
\hline NIBR-P3448 & CYPRINIDAE & Aphyocypris & chinensis & 왜몰개 & 2008-10-09 & BJ Kim \\
\hline NIBR-P3449 & ODONTOBUTIDAE & Odontobutis & interrupta & 얼록동사리 & 2008-10-09 & BJ Kim \\
\hline NIBR-P3450 & CYPRINIDAE & Zacco & platypus & 피라미 & 2008-10-09 & BJ Kim \\
\hline NIBR-P3451 & CYPRINIDAE & Acheilognathus & yamatsutae & 줄납자루 & 2008-10-09 & BJ Kim \\
\hline NIBR-P3452 & CYPRINIDAE & Microphysogobio & yaluensis & 돌마자 & 2008-10-09 & BJ Kim \\
\hline NIBR-P3453 & CENTROPOMIDAE & Coreoperca & herzi & 꺽지 & 2008-10-09 & BJ Kim \\
\hline NIBR-P3454 & CYPRINIDAE & Pungtungia & herzi & 돌고기 & 2008-10-09 & BJ Kim \\
\hline NIBR-P3455 & CYPRINIDAE & Pungtungia & herzi & 돌고기 & 2008-10-09 & BJ Kim \\
\hline NIBR-P3456 & CYPRINIDAE & Gobiobotia & brevibarba & 돌상어 & 2008-10-09 & BJ Kim \\
\hline NIBR-P3457 & CYPRINIDAE & Sarcocheilichthys & varieatus & 참중고기 & 2008-10-09 & BJ Kim \\
\hline NIBR-P3791 & CYPRINIDAE & Zacco & koreanus & 참갈겨니 & 2008-11-04 & BJ Kim \\
\hline NIBR-P3792 & CYPRINIDAE & Zacco & platypus & 피라미 & 2008-11-04 & BJ Kim \\
\hline NIBR-P3793 & CYPRINIDAE & Carassius & auratus & 붕어 & 2008-11-04 & BJ Kim \\
\hline NIBR-P3794 & COBITIDAE & Iksookimia & koreensis & 참종개 & 2008-11-04 & BJ Kim \\
\hline NIBR-P3795 & CYPRINIDAE & Pseudorasbora & parva & 참붕어 & 2008-11-04 & BJ Kim \\
\hline NIBR-P3796 & GOBIIDAE & Rhinogobius & brunneus & 밀어 & 2008-11-04 & BJ Kim \\
\hline NIBR-P3797 & CYPRINIDAE & Ladislavia & taczanowski & 새미 & 2008-11-04 & BJ Kim \\
\hline NIBR-P3798 & CYPRINIDAE & Pungtungia & herzi & 돌고기 & 2008-11-04 & BJ Kim \\
\hline NIBR-P3799 & CYPRINIDAE & Rhynchocypris & oxycephalus & 버들치 & 2008-11-04 & BJ Kim \\
\hline NIBR-P3800 & CYPRINIDAE & Rhynchocypris & steindachneri & 버들개 & 2008-11-04 & BJ Kim \\
\hline NIBR-P3801 & CENTROPOMIDAE & Coreoperca & herzi & 꺽지 & 2008-11-05 & BJ Kim \\
\hline NIBR-P3802 & CYPRINIDAE & Pungtungia & herzi & 돌고기 & 2008-11-05 & BJ Kim \\
\hline NIBR-P3803 & CYPRINIDAE & Pseudogobio & esocinus & 모래무지 & 2008-11-05 & BJ Kim \\
\hline NIBR-P3804 & CYPRINIDAE & Zacco & koreanus & 참갈겨니 & $2008-11-05$ & BJ Kim \\
\hline NIBR-P3805 & CYPRINIDAE & Zacco & platypus & 피라미 & 2008-11-05 & BJ Kim \\
\hline NIBR-P3806 & COBITIDAE & Misgurnus & anguillicaudatus & 미꾸리 & $2008-11-05$ & BJ Kim \\
\hline NIBR-P3807 & CYPRINIDAE & Pungtungia & herzi & 돌고기 & $2008-11-05$ & BJ Kim \\
\hline NIBR-P3808 & CYPRINIDAE & Acheilognathus & rhombeus & 납지리 & 2008-11-05 & BJ Kim \\
\hline NIBR-P3809 & CYPRINIDAE & Acheilognathus & lanceolatus & 납자루 & $2008-11-05$ & BJ Kim \\
\hline NIBR-P3810 & CYPRINIDAE & Coreoleuciscus & splendidus & 쉬리 & $2008-11-05$ & BJ Kim \\
\hline NIBR-P3811 & CYPRINIDAE & Carassius & auratus & 붕어 & 2008-11-05 & BJ Kim \\
\hline NIBR-P3812 & CYPRINIDAE & Carassius & auratus & 붕어 & 2008-11-05 & BJ Kim \\
\hline NIBR-P3813 & ODONTOBUTIDAE & Odontobutis & interrupta & 얼록동사리 & 2008-11-05 & BJ Kim \\
\hline NIBR-P3814 & SILURIDAE & Silurus & asotus & 메기 & 2008-11-05 & BJ Kim \\
\hline NIBR-P3815 & BAGRIDAE & Pseudobagrus & koreanus & 눈동자개 & $2008-11-05$ & BJ Kim \\
\hline NIBR-P3816 & CYPRINIDAE & Acheilognathus & rhombeus & 납지리 & $2008-11-05$ & BJ Kim \\
\hline NIBR-P3817 & CYPRINIDAE & Rhynchocypris & oxycephalus & 버들치 & $2008-11-05$ & BJ Kim \\
\hline NIBR-P3818 & CYPRINIDAE & Pungtungia & herzi & 돌고기 & $2008-11-05$ & BJ Kim \\
\hline NIBR-P3819 & CYPRINIDAE & Hemibarbus & longirostris & 참마자 & 2008-11-05 & BJ Kim \\
\hline NIBR-P3820 & CYPRINIDAE & Abbottina & rivularis & 버들매치 & 2008-11-05 & BJ Kim \\
\hline NIBR-P3821 & CYPRINIDAE & Zacco & koreanus & 참갈겨니 & 2008-11-05 & BJ Kim \\
\hline NIBR-P3822 & CYPRINIDAE & Zacco & platypus & 피라미 & 2008-11-05 & BJ Kim \\
\hline NIBR-P3823 & CYPRINIDAE & Coreoleuciscus & splendidus & 쉬리 & 2008-11-05 & BJ Kim \\
\hline NIBR-P3824 & CYPRINIDAE & Acheilognathus & lanceolatus & 납자루 & 2008-11-05 & BJ Kim \\
\hline NIBR-P3825 & CYPRINIDAE & Carassius & auratus & 붕어 & $2008-11-05$ & BJ Kim \\
\hline NIBR-P3826 & CYPRINIDAE & Zacco & koreanus & 참갈겨니 & 2008-11-05 & BJ Kim \\
\hline NIBR-P3827 & CYPRINIDAE & Zacco & platypus & 피라미 & $2008-11-05$ & BJ Kim \\
\hline NIBR-P3828 & ODONTOBUTIDAE & Odontobutis & interrupta & 얼록동사리 & 2008-11-05 & BJ Kim \\
\hline NIBR-P3829 & CYPRINIDAE & Acheilognathus & lanceolatus & 납자루 & 2008-11-05 & BJ Kim \\
\hline NIBR-P3830 & COBITIDAE & Iksookimia & koreensis & 참종개 & 2008-11-05 & BJ Kim \\
\hline NIBR-P3831 & CYPRINIDAE & Carassius & auratus & 붕어 & 2008-11-06 & BJ Kim \\
\hline NIBR-P3832 & CYPRINIDAE & Pseudogobio & esocinus & 모래무지 & 2008-11-06 & BJ Kim \\
\hline NIBR-P3833 & CYPRINIDAE & Rhynchocypris & oxycephalus & 버들치 & 2008-11-06 & BJ Kim \\
\hline NIBR-P3834 & CYPRINIDAE & Zacco & platypus & 피라미 & 2008-11-06 & BJ Kim \\
\hline NIBR-P3835 & CYPRINIDAE & Coreoleuciscus & splendidus & 쉬리 & 2008-11-06 & BJ Kim \\
\hline
\end{tabular}


Appendix 1. Continued.

\begin{tabular}{lllllll}
\hline Collection no. & \multicolumn{1}{c}{ Family } & \multicolumn{1}{c}{ Genus } & \multicolumn{1}{c}{ Species } & Korean name & Date & Collecter \\
\hline NIBR-P3836 & CYPRINIDAE & Acheilognathus & rhombeus & 납지리 & 2008-11-06 & BJ Kim \\
NIBR-P3837 & CYPRINIDAE & Hemibarbus & labeo & 누치 & 2008-11-06 & BJ Kim \\
NIBR-P3838 & CYPRINIDAE & Pseudorasbora & parva & 참붕어 & 2008-11-06 & BJ Kim \\
NIBR-P3839 & CYPRINIDAE & Abbottina & rivularis & 버들매치 & 2008-11-06 & BJ Kim \\
NIBR-P3840 & CYPRINIDAE & Hemibarbus & longirostris & 참마자 & $2008-11-06$ & BJ Kim \\
NIBR-P3841 & CYPRINIDAE & Pungtungia & herzi & 돌고기 & $2008-11-06$ & BJ Kim \\
NIBR-P3842 & CENTROPOMIDAE & Coreoperca & herzi & 꺽지 & $2008-11-06$ & BJ Kim \\
NIBR-P3843 & COBITIDAE & Misgurnus & mizolepis & 미꾸라지 & $2008-11-06$ & BJ Kim \\
NIBR-P3844 & CYPRINIDAE & Coreoleuciscus & splendidus & 쉬리 & 2008-11-06 & BJ Kim \\
NIBR-P3845 & CYPRINIDAE & Zacco & koreanus & 참갈겨니 & 2008-11-06 & BJ Kim \\
\hline
\end{tabular}

Appendix 2. Collection list of amphibians.

\begin{tabular}{|c|c|c|c|c|c|c|}
\hline Collection no. & Family & Genus & Species & Korean name & Date & Collecter \\
\hline NIBRAM100106 & BUFONIDAE & Bufo & bufo & 두꺼비 & 2008-03-18 & Sang-Cheol Lee \\
\hline NIBRAM100107 & BUFONIDAE & Bufo & bufo & 두꺼비 & 2008-03-18 & Sang-Cheol Lee \\
\hline NIBRAM100264 & HYLIDAE & Hyla & japonica & 청개구리 & $2008-05-16$ & Sang-Cheol Lee \\
\hline NIBRAM100265 & DISCOGLOSSIDAE & Bombina & orientalis & 무당개구리 & $2008-05-16$ & Sang-Cheol Lee \\
\hline NIBRAM100266 & DISCOGLOSSIDAE & Bombina & orientalis & 무당개구리 & $2008-05-16$ & Sang-Cheol Lee \\
\hline NIBRAM100267 & DISCOGLOSSIDAE & Bombina & orientalis & 무당개구리 & $2008-05-16$ & Sang-Cheol Lee \\
\hline NIBRAM100268 & DISCOGLOSSIDAE & Bombina & orientalis & 무당개구리 & $2008-05-16$ & Sang-Cheol Lee \\
\hline NIBRAM100269 & DISCOGLOSSIDAE & Bombina & orientalis & 무당개구리 & $2008-05-16$ & Sang-Cheol Lee \\
\hline NIBRAM100273 & HYNOBIIDAE & Onychodactylus & fischeri & 꼬리치레도롱뇽 & $2008-05-16$ & Sang-Cheol Lee \\
\hline NIBRAM100293 & RANIDAE & Rana & temporaria & 북방산개구리 & $2008-05-16$ & Sang-Cheol Lee \\
\hline NIBRAM100276 & BUFONIDAE & Bufo & bufo & 두꺼비 & $2008-05-22$ & Sang-Cheol Lee \\
\hline NIBRAM100277 & HYLIDAE & Hyla & japonica & 청개구리 & $2008-05-22$ & Sang-Cheol Lee \\
\hline NIBRAM100279 & HYNOBIIDAE & Onychodactylus & fischeri & 꼬리치레도롱뇽 & $2008-05-23$ & Sang-Cheol Lee \\
\hline NIBRAM100237 & RANIDAE & Pelophylax & nigromaculatus & 참개구리 & 2008-06-03 & Sang-Cheol Lee \\
\hline NIBRAM100245 & BUFONIDAE & Bufo & stejnegeri & 물두꺼비 & $2008-06-23$ & Sang-Cheol Lee \\
\hline NIBRAM100246 & HYNOBIIDAE & Onychodactylus & fischeri & 꼬리치레도롱뇽 & $2008-06-23$ & Sang-Cheol Lee \\
\hline NIBRAM100289 & RANIDAE & Rana & nigromaculata & 참개구리 & $2008-07-16$ & Sang-Cheol Lee \\
\hline NIBRAM100244 & RANIDAE & Pelophylax & nigromaculatus & 참개구리 & $2008-07-18$ & Sang-Cheol Lee \\
\hline NIBRAM100248 & HYLIDAE & Hyla & japonica & 청개구리 & $2008-07-21$ & Sang-Cheol Lee \\
\hline NIBRAM100249 & RANIDAE & Rana & rugosa & 옴개구리 & $2008-07-21$ & Sang-Cheol Lee \\
\hline NIBRAM100235 & BUFONIDAE & Bufo & stejnegeri & 물두꺼비 & 2008-09-10 & Sang-Cheol Lee \\
\hline NIBRAM100236 & RANIDAE & Pelophylax & nigromaculatus & 참개구리 & 2008-09-10 & Sang-Cheol Lee \\
\hline
\end{tabular}

Appendix 3. Collection list of reptiles.

\begin{tabular}{|c|c|c|c|c|c|c|}
\hline Collection no. & Family & Genus & Species & Korean name & Date & Collecter \\
\hline NIBRRP100158 & COLUBRIDAE & Rabdophius & tigrinus & 유혈목이 & $2008-05-17$ & Sang-Cheol Lee \\
\hline NIBRRP100162 & COLUBRIDAE & Elaphes & dione & 누룩뱀 & 2008-05-17 & Sang-Cheol Lee \\
\hline NIBRRP100163 & COLUBRIDAE & Elaphes & dione & 누룩뱀 & 2008-05-17 & Sang-Cheol Lee \\
\hline NIBRRP100154 & VIPERIDAE & Gloydius & ussuriensis & 쇠살모사 & $2008-05-20$ & Sang-Cheol Lee \\
\hline NIBRRP100155 & VIPERIDAE & Gloydius & ussuriensis & 쇠살모사 & $2008-05-20$ & Sang-Cheol Lee \\
\hline NIBRRP100164 & COLUBRIDAE & Elaphes & rufodorsata & 무자치 & 2008-05-20 & Sang-Cheol Lee \\
\hline NIBRRP100165 & COLUBRIDAE & Rabdophius & tigrinus & 유혈목이 & $2008-05-23$ & Sang-Cheol Lee \\
\hline NIBRRP100167 & VIPERIDAE & Gloydius & ussuriensis & 쇠살모사 & $2008-05-23$ & Sang-Cheol Lee \\
\hline NIBRRP100177 & COLUBRIDAE & Amphiesma & vibakari & 대륙유혈목이 & $2008-05-23$ & Sang-Cheol Lee \\
\hline NIBRRP100183 & VIPERIDAE & Gloydius & ussuriensis & 쇠살모사 & 2008-07-18 & Sang-Cheol Lee \\
\hline NIBRRP100184 & VIPERIDAE & Gloydius & ussuriensis & 쇠살모사 & 2008-07-18 & Sang-Cheol Lee \\
\hline NIBRRP100185 & VIPERIDAE & Gloydius & ussuriensis & 쇠살모사 & 2008-07-18 & Sang-Cheol Lee \\
\hline NIBRRP100156 & COLUBRIDAE & Elaphes & rufodorsata & 무자치 & 2008-07-22 & Sang-Cheol Lee \\
\hline NIBRRP100157 & COLUBRIDAE & Rabdophius & tigrinus & 유혈목이 & 2008-07-22 & Sang-Cheol Lee \\
\hline NIBRRP100159 & COLUBRIDAE & Elaphes & dione & 누룩뱀 & $2008-07-22$ & Sang-Cheol Lee \\
\hline NIBRRP100160 & VIPERIDAE & Gloydius & ussuriensis & 쇠살모사 & 2008-07-22 & Sang-Cheol Lee \\
\hline NIBRRP100169 & COLUBRIDAE & Elaphes & rufodorsata & 무자치 & 2008-10-06 & Sang-Cheol Lee \\
\hline NIBRRP100171 & COLUBRIDAE & Elaphes & dione & 누룩뱀 & 2008-10-06 & Sang-Cheol Lee \\
\hline
\end{tabular}


Appendix 4. Collection list of birds.

\begin{tabular}{|c|c|c|c|c|c|c|}
\hline Collection no. & Family & Genus & Species & Korean name & Date & Collecter \\
\hline NIBRAV100850 & PARIDAE & Parus & palustris & 쇠박새 & 2008-02-21 & Hur, Wee-Haeng \\
\hline NIBRAV100569 & TURDIDAE & Phoenicurus & auroreus & 딱새 & $2008-02-22$ & Hur, Wee-Haeng \\
\hline NIBRAV100570 & EMBERIZIDAE & Emberiza & elegans & 노랑턱멧새 & 2008-02-22 & Hur, Wee-Haeng \\
\hline NIBRAV100848 & PARIDAE & Parus & major & 박새 & 2008-03-20 & Yeong-Seok Jo \\
\hline NIBRAV100849 & PARIDAE & Parus & major & 박새 & 2008-03-20 & Yeong-Seok Jo \\
\hline NIBRAV100851 & SYLVIIDAE & Urosphena & squameiceps & 숲새 & 2008-05-15 & WH Hur, H. Seomun \\
\hline NIBRAV100799 & TURDIDAE & Phoenicurus & auroreus & 딱새 & $2008-10-22$ & WH Hur, H. Seomun \\
\hline NIBRAV100800 & TURDIDAE & Phoenicurus & auroreus & 딱새 & $2008-10-22$ & WH Hur, H. Seomun \\
\hline NIBRAV100801 & EMBERIZIDAE & Emberiza & elegans & 노랑턱멧새 & 2008-10-22 & WH Hur, H. Seomun \\
\hline NIBRAV100802 & EMBERIZIDAE & Emberiza & tristrami & 흰배멧새 & $2008-10-22$ & WH Hur, H. Seomun \\
\hline NIBRAV100803 & TURDIDAE & Phoenicurus & auroreus & 딱새 & $2008-10-22$ & WH Hur, H. Seomun \\
\hline NIBRAV100804 & EMBERIZIDAE & Emberiza & elegans & 노랑턱멧새 & $2008-10-22$ & WH Hur, H. Seomun \\
\hline NIBRAV100805 & EMBERIZIDAE & Emberiza & elegans & 노랑턱멧새 & 2008-10-22 & WH Hur, H. Seomun \\
\hline NIBRAV100806 & PARIDAE & Parus & major & 박새 & $2008-10-22$ & WH Hur, H. Seomun \\
\hline NIBRAV100807 & EMBERIZIDAE & Emberiza & elegans & 노랑턱멧새 & 2008-10-22 & WH Hur, H. Seomun \\
\hline NIBRAV100808 & EMBERIZIDAE & Emberiza & elegans & 노랑턱멧새 & $2008-10-22$ & WH Hur, H. Seomun \\
\hline NIBRAV100809 & EMBERIZIDAE & Emberiza & elegans & 노랑턱멧새 & $2008-10-22$ & WH Hur, H. Seomun \\
\hline NIBRAV100810 & EMBERIZIDAE & Emberiza & elegans & 노랑턱멧새 & $2008-10-22$ & WH Hur, H. Seomun \\
\hline NIBRAV100811 & EMBERIZIDAE & Emberiza & elegans & 노랑턱멧새 & $2008-10-22$ & WH Hur, H. Seomun \\
\hline NIBRAV100812 & TURDIDAE & Phoenicurus & auroreus & 딱새 & $2008-10-22$ & WH Hur, H. Seomun \\
\hline NIBRAV100759 & PYCNONOTIDAE & Microscelis & amaurotis & 직박구리 & $2008-10-23$ & WH Hur, H. Seomun \\
\hline NIBRAV100813 & EMBERIZIDAE & Emberiza & elegans & 노랑턱멧새 & $2008-10-23$ & WH Hur, H. Seomun \\
\hline NIBRAV100814 & EMBERIZIDAE & Emberiza & elegans & 노랑턱멧새 & $2008-10-23$ & WH Hur, H. Seomun \\
\hline NIBRAV100815 & EMBERIZIDAE & Emberiza & elegans & 노랑턱멧새 & $2008-10-23$ & WH Hur, H. Seomun \\
\hline NIBRAV100816 & EMBERIZIDAE & Emberiza & elegans & 노랑턱멧새 & $2008-10-23$ & WH Hur, H. Seomun \\
\hline NIBRAV100817 & EMBERIZIDAE & Emberiza & spodocephala & 촉새 & $2008-10-23$ & WH Hur, H. Seomun \\
\hline NIBRAV100818 & EMBERIZIDAE & Emberiza & elegans & 노랑턱멧새 & $2008-10-23$ & WH Hur, H. Seomun \\
\hline NIBRAV100819 & EMBERIZIDAE & Emberiza & elegans & 노랑턱멧새 & $2008-10-23$ & WH Hur, H. Seomun \\
\hline NIBRAV100820 & EMBERIZIDAE & Emberiza & elegans & 노랑턱멧새 & $2008-10-23$ & WH Hur, H. Seomun \\
\hline NIBRAV100821 & EMBERIZIDAE & Emberiza & elegans & 노랑턱멧새 & $2008-10-23$ & WH Hur, H. Seomun \\
\hline NIBRAV100822 & EMBERIZIDAE & Emberiza & elegans & 노랑턱멧새 & $2008-10-23$ & WH Hur, H. Seomun \\
\hline NIBRAV100823 & EMBERIZIDAE & Emberiza & elegans & 노랑턱멧새 & $2008-10-23$ & WH Hur, H. Seomun \\
\hline NIBRAV100824 & EMBERIZIDAE & Emberiza & elegans & 노랑턱멧새 & $2008-10-23$ & WH Hur, H. Seomun \\
\hline NIBRAV100825 & EMBERIZIDAE & Emberiza & elegans & 노랑턱멧새 & $2008-10-23$ & WH Hur, H. Seomun \\
\hline NIBRAV100826 & EMBERIZIDAE & Emberiza & elegans & 노랑턱멧새 & $2008-10-23$ & WH Hur, H. Seomun \\
\hline NIBRAV100827 & EMBERIZIDAE & Emberiza & elegans & 노랑턱멧새 & $2008-10-23$ & WH Hur, H. Seomun \\
\hline NIBRAV100828 & TURDIDAE & Phoenicurus & auroreus & 딱새 & $2008-10-23$ & WH Hur, H. Seomun \\
\hline NIBRAV100829 & TURDIDAE & Phoenicurus & auroreus & 딱새 & $2008-10-23$ & WH Hur, H. Seomun \\
\hline NIBRAV100830 & EMBERIZIDAE & Emberiza & elegans & 노랑턱멧새 & $2008-10-23$ & WH Hur, H. Seomun \\
\hline NIBRAV100831 & EMBERIZIDAE & Emberiza & elegans & 노랑턱멧새 & $2008-10-23$ & WH Hur, H. Seomun \\
\hline NIBRAV100832 & PARIDAE & Parus & palustris & 쇠박새 & $2008-10-23$ & WH Hur, H. Seomun \\
\hline NIBRAV100833 & PARIDAE & Parus & palustris & 쇠박새 & $2008-10-23$ & WH Hur, H. Seomun \\
\hline NIBRAV100834 & SYLVIIDAE & Phylloscopus & inornatus & 노랑눈썹솔새 & $2008-10-23$ & WH Hur, H. Seomun \\
\hline NIBRAV100835 & EMBERIZIDAE & Emberiza & elegans & 노랑턱멧새 & $2008-10-23$ & WH Hur, H. Seomun \\
\hline NIBRAV100836 & EMBERIZIDAE & Emberiza & elegans & 노랑턱멧새 & $2008-10-23$ & WH Hur, H. Seomun \\
\hline NIBRAV100837 & EMBERIZIDAE & Emberiza & spodocephala & 촉새 & $2008-10-23$ & WH Hur, H. Seomun \\
\hline NIBRAV100838 & EMBERIZIDAE & Emberiza & rustica & 쑥새 & $2008-10-23$ & WH Hur, H. Seomun \\
\hline NIBRAV100839 & PARIDAE & Parus & major & 박새 & $2008-10-23$ & WH Hur, H. Seomun \\
\hline NIBRAV100840 & PARIDAE & Parus & major & 박새 & $2008-10-23$ & WH Hur, H. Seomun \\
\hline NIBRAV100841 & PARIDAE & Parus & major & 박새 & $2008-10-23$ & WH Hur, H. Seomun \\
\hline NIBRAV100842 & PARIDAE & Parus & major & 박새 & $2008-10-23$ & WH Hur, H. Seomun \\
\hline
\end{tabular}

Appendix 5. Collection list of mammals.

\begin{tabular}{llllrrr}
\hline Collection no. & Family & Genus & Species & Korean name & Date & Collecter \\
\hline NIBRMM100728 & MURIDAE & Apodemus & agrarius & 등줄쥐 & 2008-10-07 & Yeong-Seok Jo \\
NIBRMM100729 & MURIDAE & Apodemus & agrarius & 등줄쥐 & 2008-06-26 & Yeong-Seok Jo \\
NIBRMM100730 & MURIDAE & Apodemus & agrarius & 등줄쥐 & 2008-06-26 & Yeong-Seok Jo \\
NIBRMM100731 & MURIDAE & Apodemus & agrarius & 등줄쥐 & 2008-06-26 & Yeong-Seok Jo \\
NIBRMM100732 & MURIDAE & Apodemus & agrarius & 등줄쥐 & 2008-06-26 & Yeong-Seok Jo \\
NIBRMM100733 & MURIDAE & Apodemus & agrarius & 등줄쥐 & 2008-06-26 & Yeong-Seok Jo \\
\hline
\end{tabular}


Appendix 5. Continued.

\begin{tabular}{|c|c|c|c|c|c|c|}
\hline Collection no. & Family & Genus & Species & Korean name & Date & Collecter \\
\hline NIBRMM100734 & MURIDAE & Apodemus & agrarius & 등줄쥐 & $2008-06-26$ & Yeong-Seok Jo \\
\hline NIBRMM100735 & MURIDAE & Apodemus & agrarius & 등줄쥐 & 2008-06-26 & Yeong-Seok Jo \\
\hline NIBRMM100736 & MURIDAE & Apodemus & agrarius & 등줄쥐 & $2008-06-26$ & Yeong-Seok Jo \\
\hline NIBRMM100737 & MURIDAE & Apodemus & agrarius & 등줄쥐 & 2008-06-26 & Yeong-Seok Jo \\
\hline NIBRMM100752 & MURIDAE & Apodemus & agrarius & 등줄쥐 & 2008-03-18 & Yeong-Seok Jo \\
\hline NIBRMM100754 & CERVIDAE & Hydropotes & inermis & 고라니 & 2008-03-18 & Yeong-Seok Jo \\
\hline NIBRMM100755 & CERVIDAE & Hydropotes & inermis & 고라니 & 2008-03-18 & Yeong-Seok Jo \\
\hline NIBRMM100756 & MURIDAE & Mus & musculus & 생쥐 & 2008-03-19 & Yeong-Seok Jo \\
\hline NIBRMM100757 & MURIDAE & Apodemus & agrarius & 등줄쥐 & 2008-03-19 & Yeong-Seok Jo \\
\hline NIBRMM100758 & MURIDAE & Apodemus & agrarius & 등줄쥐 & 2008-03-19 & Yeong-Seok Jo \\
\hline NIBRMM100759 & MURIDAE & Apodemus & agrarius & 등줄쥐 & 2008-03-19 & Yeong-Seok Jo \\
\hline NIBRMM100760 & SCIURIDAE & Tamias & sibiricus & 다람쥐 & 2008-03-20 & Yeong-Seok Jo \\
\hline NIBRMM100761 & MURIDAE & Apodemus & agrarius & 등줄쥐 & 2008-03-20 & Yeong-Seok Jo \\
\hline NIBRMM100762 & MURIDAE & Apodemus & agrarius & 등줄쥐 & 2008-03-20 & Yeong-Seok Jo \\
\hline NIBRMM100764 & MURIDAE & Apodemus & agrarius & 등줄쥐 & 2008-03-20 & Yeong-Seok Jo \\
\hline NIBRMM100765 & MURIDAE & Apodemus & agrarius & 등줄쥐 & 2008-03-21 & Yeong-Seok Jo \\
\hline NIBRMM100766 & MURIDAE & Apodemus & agrarius & 등줄쥐 & 2008-03-21 & Yeong-Seok Jo \\
\hline NIBRMM100807 & MURIDAE & Apodemus & agrarius & 등줄쥐 & 2008-10-07 & Yeong-seok Jo \\
\hline NIBRMM100808 & MURIDAE & Apodemus & agrarius & 등줄쥐 & 2008-10-07 & Yeong-seok Jo \\
\hline NIBRMM100809 & MURIDAE & Apodemus & agrarius & 등줄쥐 & 2008-10-07 & Yeong-seok Jo \\
\hline NIBRMM100810 & MURIDAE & Apodemus & agrarius & 등줄쥐 & 2008-10-07 & Yeong-seok Jo \\
\hline NIBRMM100811 & MURIDAE & Apodemus & agrarius & 등줄쥐 & 2008-10-07 & Yeong-seok Jo \\
\hline NIBRMM100812 & MURIDAE & Mus & musculus & 생쥐 & 2008-10-07 & Yeong-seok Jo \\
\hline NIBRMM100813 & MURIDAE & Apodemus & agrarius & 등줄쥐 & 2008-10-07 & Yeong-seok Jo \\
\hline NIBRMM100814 & MURIDAE & Apodemus & agrarius & 등줄쥐 & 2008-10-07 & Yeong-seok Jo \\
\hline NIBRMM100815 & SORICIDAE & Crocidura & lasiura & 땃쥐 & 2008-10-08 & Yeong-seok Jo \\
\hline NIBRMM100816 & MURIDAE & Apodemus & agrarius & 등줄쥐 & 2008-10-08 & Yeong-Seok Jo \\
\hline NIBRMM100817 & MURIDAE & Apodemus & agrarius & 등줄쥐 & 2008-10-08 & Yeong-Seok Jo \\
\hline NIBRMM100818 & MURIDAE & Apodemus & agrarius & 등줄쥐 & 2008-10-08 & Yeong-Seok Jo \\
\hline NIBRMM100819 & MURIDAE & Apodemus & agrarius & 등줄쥐 & 2008-10-08 & Yeong-Seok Jo \\
\hline NIBRMM100820 & MURIDAE & Apodemus & agrarius & 등줄쥐 & 2008-10-08 & Yeong-Seok Jo \\
\hline NIBRMM100821 & MURIDAE & Apodemus & agrarius & 등줄쥐 & 2008-10-08 & Yeong-Seok Jo \\
\hline NIBRMM100822 & MURIDAE & Apodemus & agrarius & 등줄쥐 & 2008-10-08 & Yeong-Seok Jo \\
\hline NIBRMM100857 & MURIDAE & Apodemus & agrarius & 등줄쥐 & 2008-06-26 & Yeong-Seok Jo \\
\hline NIBRMM100858 & MURIDAE & Apodemus & agrarius & 등줄쥐 & 2008-06-26 & Yeong-Seok Jo \\
\hline NIBRMM100859 & MURIDAE & Apodemиs & agrarius & 등줄쥐 & $2008-06-26$ & Yeong-Seok Jo \\
\hline NIBRMM100860 & MURIDAE & Apodemus & agrarius & 등줄쥐 & $2008-06-26$ & Yeong-Seok Jo \\
\hline NIBRMM100861 & MURIDAE & Apodemus & agrarius & 등줄쥐 & 2008-06-26 & Yeong-Seok Jo \\
\hline NIBRMM100862 & MURIDAE & Apodemus & agrarius & 등줄쥐 & 2008-06-26 & Yeong-Seok Jo \\
\hline NIBRMM100863 & MURIDAE & Apodemus & agrarius & 등줄쥐 & 2008-06-26 & Yeong-Seok Jo \\
\hline NIBRMM100864 & MURIDAE & Apodemus & agrarius & 등줄쥐 & 2008-06-26 & Yeong-Seok Jo \\
\hline NIBRMM100865 & MURIDAE & Apodemus & agrarius & 등줄쥐 & 2008-03-19 & Yeong-Seok Jo \\
\hline NIBRMM100866 & MURIDAE & Apodemus & agrarius & 등줄쥐 & 2008-03-20 & Yeong-Seok Jo \\
\hline NIBRMM100868 & MURIDAE & Apodemus & agrarius & 등줄쥐 & 2008-03-20 & Yeong-Seok Jo \\
\hline NIBRMM100869 & MURIDAE & Apodemus & agrarius & 등줄쥐 & 2008-03-21 & Yeong-Seok Jo \\
\hline NIBRMM100870 & MURIDAE & Apodemus & agrarius & 등줄쥐 & 2008-03-21 & Yeong-Seok Jo \\
\hline NIBRMM100889 & SCIURIDAE & Tamias & sibiricus & 다람쥐 & $2008-03-20$ & Yeong-Seok Jo \\
\hline NIBRMM100893 & MURIDAE & Apodemus & agrarius & 등줄쥐 & 2008-03-18 & Yeong-Seok Jo \\
\hline NIBRMM100894 & MURIDAE & Mus & musculus & 생쥐 & 2008-03-19 & Yeong-Seok Jo \\
\hline NIBRMM100895 & MURIDAE & Apodemus & agrarius & 등줄쥐 & 2008-03-19 & Yeong-Seok Jo \\
\hline NIBRMM100897 & MURIDAE & Apodemus & agrarius & 등줄쥐 & 2008-03-19 & Yeong-Seok Jo \\
\hline NIBRMM100899 & MURIDAE & Apodemus & agrarius & 등줄쥐 & $2008-06-26$ & Yeong-Seok Jo \\
\hline NIBRMM100900 & MURIDAE & Apodemus & agrarius & 등줄쥐 & 2008-06-26 & Yeong-Seok Jo \\
\hline NIBRMM100901 & MURIDAE & Apodemus & agrarius & 등줄쥐 & 2008-10-07 & Yeong-seok Jo \\
\hline NIBRMM100902 & MURIDAE & Apodemus & agrarius & 등줄쥐 & 2008-10-07 & Yeong-seok Jo \\
\hline NIBRMM100903 & MURIDAE & Apodemus & agrarius & 등줄쥐 & 2008-10-07 & Yeong-seok Jo \\
\hline NIBRMM100904 & MURIDAE & Apodemus & agrarius & 등줄쥐 & 2008-10-07 & Yeong-seok Jo \\
\hline NIBRMM100905 & MURIDAE & Apodemus & agrarius & 등줄쥐 & 2008-10-07 & Yeong-seok Jo \\
\hline NIBRMM100906 & MURIDAE & Apodemus & agrarius & 등줄쥐 & 2008-10-07 & Yeong-seok Jo \\
\hline NIBRMM100907 & MURIDAE & Apodemus & agrarius & 등줄쥐 & 2008-10-07 & Yeong-seok Jo \\
\hline NIBRMM100908 & MURIDAE & Apodemus & agrarius & 등줄쥐 & 2008-10-07 & Yeong-seok Jo \\
\hline NIBRMM100909 & SORICIDAE & Crocidura & lasiura & 땃쥐 & 2008-10-08 & Yeong-seok Jo \\
\hline NIBRMM100910 & MURIDAE & Apodemus & agrarius & 등줄쥐 & $2008-10-08$ & Yeong-Seok Jo \\
\hline
\end{tabular}

